\title{
Improving child and adolescent mental health care provision by scrutiny of national diagnostic practices: A call for increased international knowledge exchange.
}

\author{
B. T. H. de Veen ${ }^{1} \cdot$ J. Hebebrand ${ }^{2} \cdot$ W. G. Staal ${ }^{3}$
}

Published online: 4 October 2018

๑) Springer-Verlag GmbH Germany, part of Springer Nature 2018

It is well known that in the field of medicine, international collaborations are highly fruitful and for instance help to improve the medical care for patients and promote a more efficient organization of health care systems. These exchanges between organizations in different countries may exist over large distances, but can also be meaningful between neighboring organizations in different countries. To illustrate this we discuss the potential insights that can be obtained by the exchange of expertise and sharing of data of neighboring child- and adolescent psychiatric hospitals between Germany and the Netherlands.

Germany and the Netherlands share a high expertise in research in the field of Child and Adolescent Psychiatry. Between 2005 and 2010 Germany and the Netherlands appeared to be among the most productive countries in this field, accounting for 458 and 314 publications, respectively [1]. Furthermore, based on regulation from the European Union, Dutch and German psychiatrists are automatically recognized and qualified to work in their neighbor's country $[9,10]$. In practice, however, bureaucratic barriers for medical registration make it difficult and expensive for doctors to work across international borders. Despite fruitful research collaborations, more practical collaborations and exchange of expertise between Dutch and German child and adolescent psychiatrists remains scarce [7]. In general, international exchange programs have been shown to promote

B. T. H. de Veen

b.deveen@karakter.com

1 Karakter, Child- and Adolescent Psychiatry, Nijmegen, The Netherlands

2 University of Duisburg-Essen, Child- and Adolescent Psychiatry, Duisburg-Essen, Germany

3 Karakter Child- and Adolescent Psychiatry, Nijmegen and Radboudumc Nijmegen The Netherlands, The Faculty of Social Sciences, Leiden University, Leiden, The Netherlands medical competencies, scientific research collaborations and health innovation. As such, both countries could highly benefit from an increased cross-border exchange of knowledge and gain an improved insight in the organization of each of the health care systems, cultural expects of diagnosis and classification practices, differences in types of treatment that are provided, etc.

A striking example of this principle is the comparison of the relative prevalence of psychiatric disorders such as autism spectrum disorder (ASD) between the two countries. One would expect that the prevalence rates between these two neighboring countries would be quite similar. However, the administrative estimated prevalence (i.e. calculated prevalence of autism spectrum disorder using administrative outpatient data from a nationwide health insurance fund) of ASD diagnoses in children and adolescents in Germany was reported to be $0.6 \%$ [3], which is markedly lower than the reported estimated administrative prevalence of $1.7 \%$ for the Netherlands [4].

This marked difference is unlikely to be explained by genetic factors, since both countries are in close geographical proximity, have a long history of trade and no important natural barriers that would hinder exchange between populations. Indeed, German and Dutch populations are although slightly genetically distinctive, very closely related $[6,8]$. The differences in prevalence rates are neither likely to be explained by differences in economic wealth, whether expressed as human capital (e.g. educational level and skills) nor more physical forms of wealth, such as having a strong infrastructure. In the unlikely event that the differences are real, research would be required to elucidate environmental or non-environmental factors underlying the substantial difference.

The differences in the estimated administrative prevalence rates are most likely based on classification, service availability and awareness of ASD in both the general public as well as the medical field. Interestingly, when observing 
data from symptom dimensions based assessment with the Child Behavior Checklist (CBCL) in the general population, scores between Germany and the Netherlands appear to be quite similar [5]. This implies that the clinical presentation of psychiatric disorders in the general population seems to largely overlap between both countries, but somehow still may result in differences in classification.

Since 2015 the Netherlands has reformed its reimbursement system for the mental health care for children. The responsibility for the reimbursement of the child and adolescent psychiatric care was shifted from the health insurance companies to the municipalities to bring child and youth psychiatric and psychosocial care under one financial and administrative structure. In Germany, the reimbursement system for child and adolescent psychiatric care is based on the health insurance companies, thereby resembling the former Dutch reimbursement system. It is possible that the different reimbursement systems may be a factor in influencing administrative prevalence between countries, although in the Netherlands the administrative prevalence rates did not change as a result of the reforms in the reimbursement system. Furthermore, both countries provide financial aid to parents with children diagnosed with mental disorders such as ASD. Presuming Dutch and German psychiatric care for children is similarly well provided for, this raised the question whether there is a cultural difference on the level of medical practitioners. For example, in Germany pediatricians (subspecialty "social pediatrics") see young children with developmental problems more often than child and adolescent psychiatrists. As a result, the diagnosis of ASD is often made by those pediatricians who are specialized in "social pediatrics. In contrast, in the Netherlands the official diagnosis of ASD is a core domain of child and adolescent psychiatry.

On an organizational level, preliminary investigations by the Dutch academic child and adolescent psychiatric institution Karakter, and the German academic LVR clinic at the University of Duisburg-Essen found differences in how mental care is organized and the different care models being employed. For example, in Germany there is predominantly inpatient care, whereas in the Netherlands the vast majority is outpatient care (e.g. through Intensive Home Treatment). Mutually comparing data on practical and theoretical approaches to providing mental care may complement organizations profiles thereby greatly benefiting both sides. On a clinical level, preliminary investigations revealed a large difference in reported administrative prevalence. Significant differences in reported administrative prevalence rates were found for ASD and ADHD, which were both higher for Karakter, and depressive disorders, being higher for the LVR clinic. In part, this difference may reflect differences in service provision. Thus, as stated above in Germany children with ADHD and/or
ASD are frequently diagnosed and treated by pediatricians (and in particular social pediatricians).

Indeed, differences in referral patterns, health care network and sub-specializations of different institutions will influence patient populations. However, preliminary results of an additional exchange program with highly trained professionals from the participating institutes in the field of child and adolescent psychiatry confirmed a difference in perception at the level of individual patients. This was especially the case in patients with ASD and affective disorder symptoms with Dutch experts more frequently endorsing the diagnosis of ASD than the German professionals (and vice versa for affective disorders). For example, adolescents, who were socially withdrawn within the context of school refusal, were more likely to be diagnosed as having major depression or social phobia by the colleagues in Essen, whereas the diagnosis of ASD was considered more frequently by the Dutch experts. Considering that both neighboring professionals are highly trained and experienced in the field of psychiatric diagnostics at certified institutions, this discrepancy in assessment is very unlikely to be explained by a difference in level of education or training. Rather, cultural factors and different work methods may influence assessment of psychiatric disorders. One example relates to the speed of the diagnostic assessment; whereas two-three appointments are the rule in Essen, the working diagnosis/diagnoses in Karakter is/are reached at the initial presentation.

The importance of such cultural factors in diagnosing is underlined by the incorporation of cultural sensitivity in the DSM-5 [2]. The description is aimed to help medical professionals better diagnose patients with a different cultural background. However, this fails to address the issue of cross-cultural differences in perspectives of professionals on mental disorders. It is not implied that medical professionals do not recognize symptomatic behavior, but rather that it is categorized differently. This raises many additional questions. For instance, what are the implications for treatment effectiveness when patients are differently categorized? Should treatment be based on classification, or rather on dimensional symptom approaches? Can interventions be developed that account for cultural differences between countries?

We call for an increased international knowledge exchange not only between scholars, but also between medical professionals at the level of clinical practice, learning from existing treatment programs and identifying common factors that predict improvement in different countries. Clinical research should highly benefit from such collaborations, especially when this involves building bridges between scholars, clinicians and patients and their caregivers. 


\section{Compliance with ethical standards}

Conflict of interest The authors declare that they have no conflict of interest.

\section{References}

1. Albayrak O, Focker M, Wibker K, Hebebrand J (2012) Bibliometric assessment of publication output of child and adolescent psychiatric/psychological affiliations between 2005 and 2010 based on the databases PubMed and Scopus. Eur Child Adolesc Psychiatry 21(6):327-337

2. APA (2013) Diagnostic and statistical manual of mental disorders, 5th edn. APA, Washington, DC

3. Bachmann C, Gerste B, Hoffmann F (2018) Diagnoses of autism spectrum disorders in Germany: time trends in administrative prevalence and diagnostic stability. Autism 22(3):283-290

4. CBS (2012) CBS Statline, ASD diagnoses 2012. Centraal Bureau voor de Statistiek, Voorburg

5. Crijnen A, Achenbach T, Verhulst F (1997) Comparisons of problems reported by parents of children in 12 cultures: total problems, externalizing, and internalizing. J Am Acad Child Adolesc Psychiatry 36(9):1269-1277

6. Kayser M, Krawczak M, Excoffier L, Dieltjes P, Corach D, Pascali V, de Knijff P (2001) An extensive analysis of Y-chromosomal microsatellite haplotypes in globally dispersed human populations. Am J Hum Genet 68(4):990-1018

7. Revet A, Hebebrand J, Klauser P (2017) The 2017 ESCAP Research Academy Workshop: bright perspectives for child and adolescent psychiatry. Eur Child Adolesc Psychiatry 26(10):1279-1280

8. Roewer L, Kayser M, Dieltjes P, Nagy M, Bakker E, Krawczak M, de Knijff P (1996) Analysis of molecular variance (AMOVA) of Y-chromosome-specific microsatellites in two closely related human populations. Hum Mol Genet 5(7):1029-1033

9. Union E (2005) Directive 2005/36/EC of the European Parliament and of the Council of 7 September 2005 on the recognition of professional qualifications

10. Union E (2013) Directive 2013/55/EU of the European Parliament and of the Council of 20 November 2013 amending Directive 2005/36/EC on the recognition of professional qualifications and Regulation (EU) No. 1024/2012 on administrative cooperation through the Internal Market Information System ('the IMI Regulation') 